\title{
Measuring Conductance of Phenylenediamine as a Molecular Sensor
}

\author{
Taekyeong Kim ${ }^{1}$ and Tae Hyun Kim ${ }^{2}$ \\ ${ }^{1}$ Department of Physics, Hankuk University of Foreign Studies, Yongin 449-791, Republic of Korea \\ ${ }^{2}$ Department of Chemistry, Soonchunhyang University, Asan 336-745, Republic of Korea
}

Correspondence should be addressed to Tae Hyun Kim; thkim@sch.ac.kr

Received 24 March 2015; Revised 4 May 2015; Accepted 4 May 2015

Academic Editor: Xiao-Miao Feng

Copyright (C) 2015 T. Kim and T. H. Kim. This is an open access article distributed under the Creative Commons Attribution License, which permits unrestricted use, distribution, and reproduction in any medium, provided the original work is properly cited.

\begin{abstract}
We report experimental measurements of molecular conductance as a single molecular sensor by using scanning tunneling microscope-based break-junction (STM-BJ) technique. The gap was created after Au atomic point contact was ruptured, and the target molecule was inserted and bonded to the top and bottom electrodes. We successfully measured the conductance for a series of amine-terminated oligophenyl molecules by forming the molecular junctions with Au electrodes. The measured conductance decays exponentially with molecular backbone length, enabling us to detect the type of molecules as a molecular sensor. Furthermore, we demonstrated reversible binary switching in a molecular junction by mechanical control of the gap between the electrodes. Since our method allows us to measure the conductance of a single molecule in ambient conditions, it should open up various practical molecular sensing applications.
\end{abstract}

\section{Introduction}

Detecting electrical properties of various molecules is crucial to the development of practical molecular sensors as well as nanoscale organic-based devices. Over the past decade, extensive efforts have been devoted to measuring the conductance of a few molecules or even a single molecule. For example, the graphene or nanotube-based molecular sensors have been used to detect the target molecules by changing the surface electrical potential of the devices with the molecules [1-6]. However, it is very difficult to functionalize the pristine graphene surface with the chemical or biological molecules due to the perfect structures of graphene. Another example is the conducting atomic force microscope (AFM) which can also be used to investigate the electrical properties of selfassembled molecules on Au substrate. However, this method involves several delicate assembly processes and it is not able to calculate the accurate number of the molecules on the Au-coated AFM probe and the substrate $[7,8]$. The scanning tunneling microscope-based break-junction (STM$\mathrm{BJ}$ ) technique is one of the most versatile techniques to create a single metal-molecule junction and detect the electrical conductance as a molecular sensor under ambient conditions [9-14]. The STM-BJ is easy to load the sample and very fast to measure the molecular conductance formed with the metal electrodes. Therefore, we can do the statistical analysis based on a lot of data sets for measurements of the molecular conductance by using the STM-BJ technique. The STM-BJ can be also combined with the AFM to demonstrate simultaneous force and conductance measurements on metal-moleculemetal junctions [15]. Furthermore, recently the molecular diode as well as switch in a single molecule level has been generated by the STM-BJ technique [16, 17]. On the other hand, this technique can be used for understanding the optical and thermal properties in a single molecule junction and investigating their influence on electronic transport in individual molecular devices $[18,19]$. In most papers of previous works, molecular sensor system needs a number of molecules to detect and characterize them; however the STMBJ can measure the conductance of a single molecule and investigate the electrical properties in a single molecule level [20-22]. Furthermore, this technique is reliable and stable in an ambient condition, which enables us to make a molecular sensor with the high resolution and the reproducibility. 


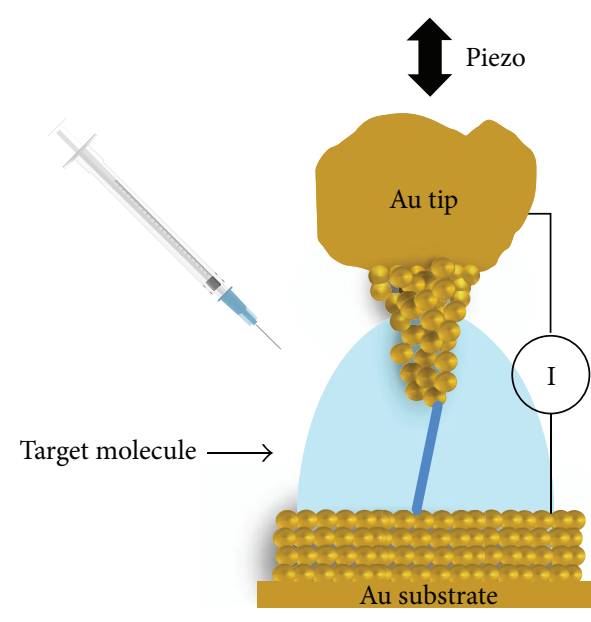

(a)

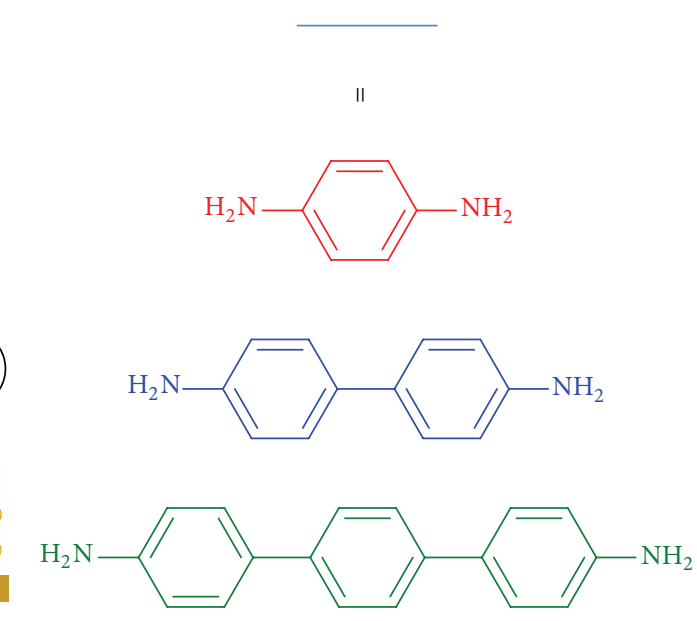

(b)

FIGURE 1: (a) Illustration of an STM-BJ setup for the conductance measurement of the target molecules in solution. (b) Structures of a series of amine-terminated oligophenyls as the target molecule with $n=1-3$ benzene groups.

In this study, we have used the STM-BJ technique to detect the target molecule which is a series of amineterminated oligophenyl derivatives and measured the electrical conductance of the molecules in ambient conditions. After the metal point contact of Au top and bottom electrodes is ruptured, the molecule can be inserted in the gap and bind onto the electrodes using an amine $\left(\mathrm{NH}_{2}\right)$ linker group. We found that the measured conductance by forming the molecule-metal junctions decreases exponentially with molecular backbone length, resulting in the detection of target molecules as a molecule sensor. We also presented the on-off binary switching in a molecular junction by mechanical control of the gap between the electrodes.

\section{Experimental}

We used a freshly cut Au wire tip (Alfa-Aesar, 99.9985\% purity, diameter $0.25 \mathrm{~mm}$ ) and Au-coated mica substrate as the top and bottom electrodes with an STM-BJ setup which operates in ambient conditions at room temperature as previously described in detail [10]. We drop the $1 \mathrm{mM}$ solution of the target molecules in 1,2,4-trichlorobenzene solution (Sigma-Aldrich 99\% Aldrich) by using the syringe on the Au-coated mica substrate for the measurements. A series of amine-terminated oligophenyl derivatives as the target molecules were obtained from Sigma-Aldrich and were used without further purification. The conductance of the molecular junction formed with the Au electrode was measured by repeatedly forming and breaking Au point contacts with an STM-BJ setup. Each conductance measurement starts by moving the tip into the substrate to create a metal point contact. It enables us to create a new electrode structure for each measurement. The tip is then retracted from the substrate at a speed of $\sim 50 \mathrm{~nm} / \mathrm{s}$, while the current is recorded at a fixed applied bias voltage of $30 \mathrm{mV}$ at a $40 \mathrm{kHz}$ data acquisition rate, enabling us to measure a conductance (current/voltage) versus displacement trace. Thousands of curves were collected for the detailed statistical analysis under the STM-BJ system. We can measure thousands of the single molecular conductance trace in ambient conditions with a short time, which is innovative in the molecular electronics. Furthermore, we have used the $\mathrm{Au}$ as the electrodes, because $\mathrm{Pt}$ is more expensive than $\mathrm{Au}$, and $\mathrm{Ag}$ is easily oxidized in ambient condition $[23,24]$.

\section{Results and Discussion}

Figure 1(a) shows the illustration of an experimental setup for the conductance measurement of the target molecules in solution. The Au tip as the top electrode was connected electrically with the Au-coated mica substrate as the bottom electrode. The target molecule in 1,2,4-trichlorobenzene solution was dropped onto Au-coated mica substrate by using syringe. We controlled the gap between top and bottom $\mathrm{Au}$ electrodes by moving a piezo in $z$-axis, and the target molecule can be bridged with the electrodes, resulting in detection of the electrical conductance of the target molecules. Figure 1(b) shows the structures of a series of amine-terminated oligophenyl derivatives as the target molecules with $n=1-3$ benzene groups.

Figure 2 shows a schematic diagram of the contact and retraction steps of the top and bottom Au electrodes which have the target molecule solution on the substrate, by moving the piezo under the STM-BJ system. The black arrow indicates the piezo movement in $z$-axis. Initially, the Au tip is approached and contacted onto the Au substrate, resulting in a conductance of at least $10 G_{0}$ (i to iii), where $G_{0}=2 e^{2} / h$ (the quantum conductance). By retraction of $\mathrm{Au}$ tip, the $\mathrm{Au}$ atomic channels are formed and decreased in the number of the channels due to the elastic relaxation of the Au atoms (iv and v). Finally the single-atomic channel (v) is ruptured in $\mathrm{Au}$ junction part during stretching of atomic-sized contacts, resulting in the gap of the Au electrode. Since the gap distance (less than $0.6 \mathrm{~nm}$ ) $[17,25]$ for $\mathrm{Au}$ is smaller than that of 

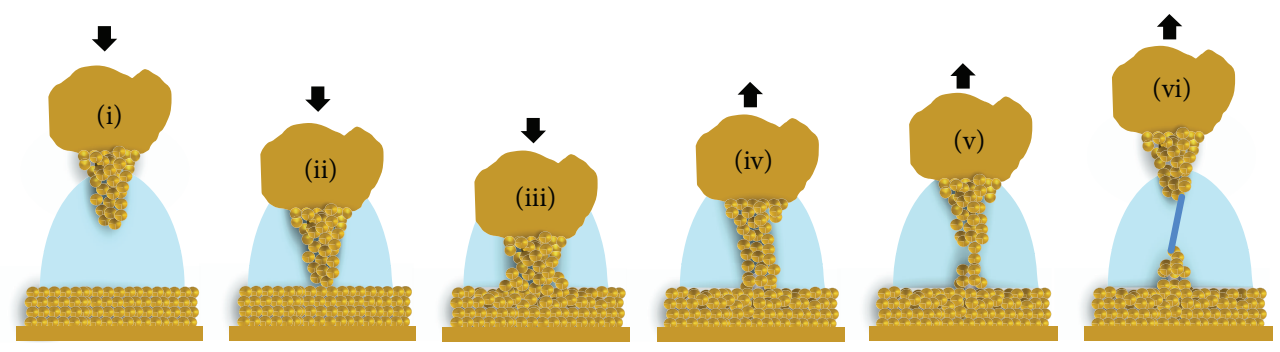

FIGURE 2: Schematic diagram for the contact and retraction steps of the top and bottom Au electrodes by using a piezo movement under the STM-BJ system. The final stage (vi) indicates the molecular junction formed with the Au electrodes in solution, resulting in the detection of the target molecule.

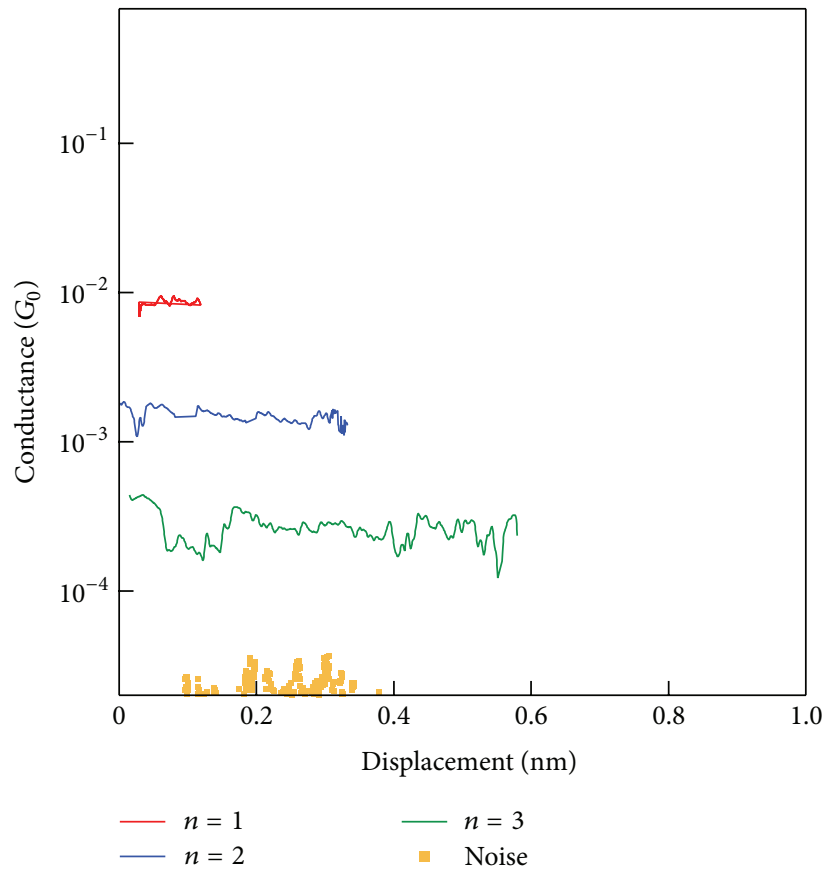

(a)

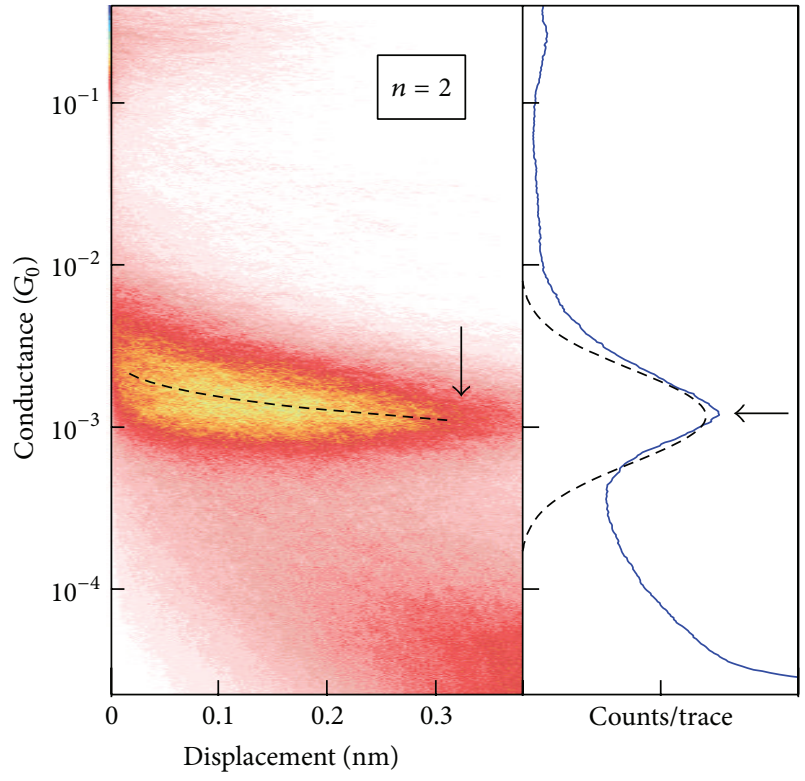

(b)

Figure 3: (a) Measured conductance trace of the target molecules, $n=1$ (red), $n=2$ (blue), $n=3$ (green), and no molecule (yellow). (b) Two-dimensional conductance-displacement histogram for target molecule $(n=2)$. The dashed line in left panel is the fitting profile which means the extended molecular length as indicated by the arrow. Histogram of the conductance (blue) is shown in left panel with a Gaussian fitting (black dashed curve). The arrow indicates the conductance peak value.

the molecular length, the molecule can be inserted in this small gap, and the metal-molecule-metal junction is formed (vi). The bonding mechanism between $\mathrm{Au}$ and the $\mathrm{NH}_{2}$ is a simple delocalization of the lone-pair of electrons from the amine nitrogen $(\mathrm{N})$ to coordinatively unsaturated surface $\mathrm{Au}$ atoms [11, 26, 27]. Finally, we can measure the conductance of the target molecule in the solution. The amine linker group forms the most stable molecular binding structures with the Au electrodes compared to other linkers such as thiol, methylsulfide, and isonitrile. For example, for thiols (S) on $\mathrm{Au}$, the bonding force for the Au-S bond is larger than that for the individual $\mathrm{Au}$ atomic bond, leading to grain migration, pitting of $\mathrm{Au}$ electrodes, and metal island formation that complicate the investigation of single molecule conductance measurements [28].
Figure 3(a) shows the measured conductance traces of the target molecules, $n=1$ (red), $n=2$ (blue), $n=3$ (green), and no molecule (yellow) by using STM-BJ technique. We observed a clear plateau at a specific conductance in the range of $\sim 10^{-2}-10^{-4} G_{0}$, which decays exponentially with molecular backbone length. It is well known that the amine-terminated molecules conduct through the highest occupied molecular orbital (HOMO). Furthermore, the HOMO resonance energy is close to the Au Fermi energy with increasing the molecular backbone. However, the short molecule is better couple to the $\mathrm{Au}$ electrodes, resulting in a higher conductance compared to the long molecules $[29,30]$. We also found that the plateau length of the molecule increases with the number of benzene rings. To probe the reproducibility of the detection of molecular conductance, we repeated 


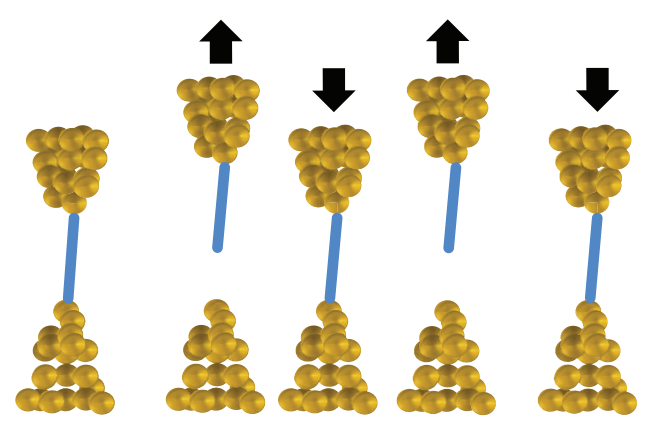

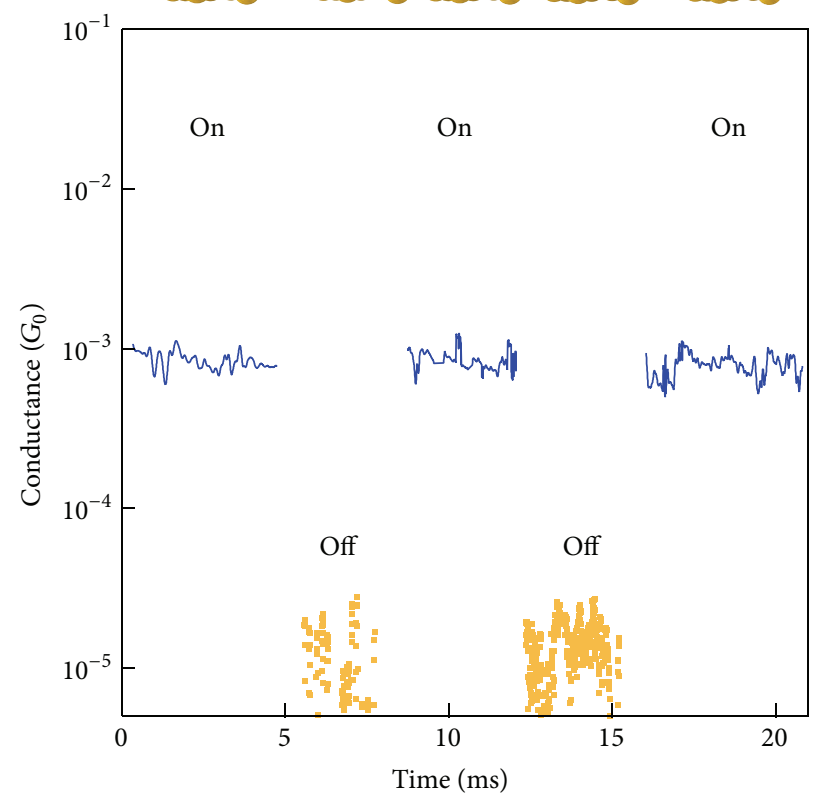

(a)

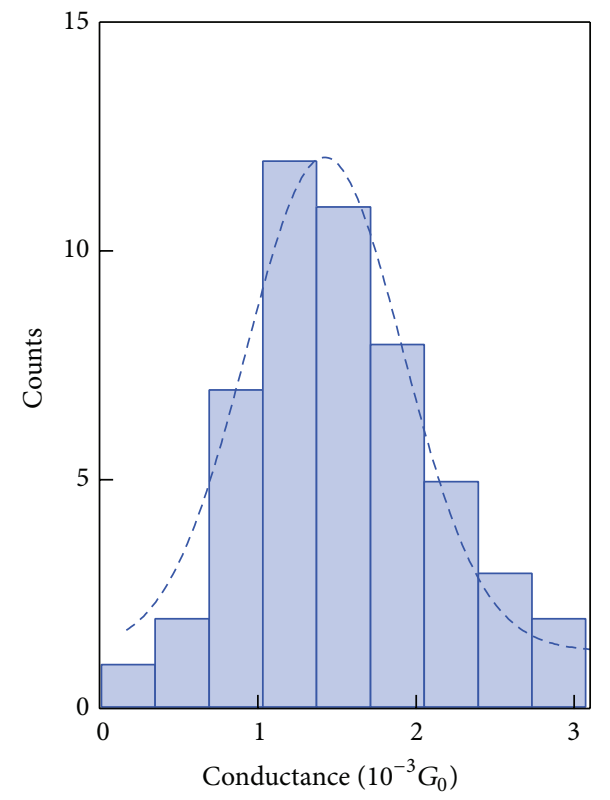

(b)

Figure 4: (a) Sample switching conductance traces with "on" and "off” states. The upper panel shows the molecular junction structures according to the piezo movements indicated by the arrow. (b) Conductance histogram generated from the average value of the conductance for each "on" state. The blue dashed curve is the Gaussian fit to the distribution.

the measurements with thousands of molecular junctions through the STM-BJ technique in the same manner. A twodimensional (2D) conductance-displacement histogram for the $4,4^{\prime}$-diaminobiphenyl molecule $(n=2)$ is generated in order to show all the traces at a glance in Figure 3(b) $[31,32]$. We overlay all measured conductance traces after aligning them along the positive displacement ( $x$-axis) at a conductance of $1 G_{0}$ because the conductance plateau occurs in random locations along the displacement axis. Logarithmic bins are used for the conductance axis and linear bins for the displacement axis. The 2D conductance-displacement histogram (with over 2,000 traces) and the conductance fitting profile (black dashed line) in the left panel show that the molecular conductance peak extends to approximately $0.3 \mathrm{~nm}$ along the $x$-axis. The arrow points to a fully extended molecular junction before breaking the bond between the molecule and the $\mathrm{Au}$ atomic electrodes. The right panel in Figure 3(b) shows the logarithmically binned conductance histogram generated without data selection for the molecule using a linear bin size of $0.0001 G_{0}$. The black dashed curve is the Gaussian fit for the conductance peak of the target molecule. The most probable molecular conductance is of $\sim 1.2 \times 10^{-3} G_{0}$ for the molecular junctions, as indicated by the arrow obtained from Gaussian fitting. This indicates that the $\mathrm{NH}_{2}$ end group of the molecule is well bonded to the Au metal atoms, resulting in a stable formation of a molecular junction as a molecular sensor.

As a proof of concept, we successfully demonstrated the reversible conductance switching of the molecular junctions by controlling the piezo. We detect the target molecule between the Au tip and the Au substrate while applying two types of ramps to the piezoelectric actuator that modulates the substrate position. Figure 4(a) is the sample switching conductance traces which clearly show the "on" and "off" states of the molecular junction (4,4' -diaminobiphenyl, $n=$ 2) according to the piezo position with $\sim 0.3 \mathrm{~nm}$ amplitude as indicated by the arrows in upper panel. The "on" state is for the molecular junction (blue in shadow box) which has the conductance of $\sim 1 \times 10^{-3} G_{0}$, and the "off" state is for the noise level (yellow) which means no molecule between the electrodes. Figure 4(b) shows the conductance histogram generated from the average value of the conductance for each "on" state. The blue dashed curve is the Gaussian fit to the distribution. The conductance peak value from 
the fit is $1.4 \times 10^{-3} G_{0}$ which is consistent with the normal measurement results in Figure 3(b). It confirms that we can make on-off binary switching in a molecular junction by mechanical control of the gap between the electrodes.

\section{Conclusion}

We detected the target molecule by bridging it to the Au electrodes through the STM-BJ technique in ambient conditions at room temperature. We successfully measured the conductance of a series of amine-terminated oligophenyl derivatives and observed that the conductance decays exponentially with the molecular backbone length. We also found that the plateau length of molecules increases with the number of benzene rings, which enables us to discriminate each other as a molecule sensor. Furthermore, we demonstrated the reversible binary molecular switching by controlling the gap of the $\mathrm{Au}$ electrodes. Our works may open a new path to the stable molecular sensors which are operated in ambient conditions and can be used for measuring the conductance in a single molecule level.

\section{Conflict of Interests}

The authors declare that there is no conflict of interests regarding the publication of this paper.

\section{Acknowledgments}

This work was supported by Basic Science Research Program through the National Research Foundation of Korea (NRF) funded by the Ministry of Education (NRF2014R1A1A2053848 and NRF-2013R1A1A2012434). This work (Grant no. C0221297) was supported by Business for Academic-Industrial Cooperative Establishments funded by Korea Small and Medium Business Administration in 2014.

\section{References}

[1] R. Stine, J. T. Robinson, P. E. Sheehan, and C. R. Tamanaha, "Real-time DNA detection using reduced graphene oxide field effect transistors," Advanced Materials, vol. 22, no. 46, pp. 52975300, 2010.

[2] T. Kim, J. Park, H. J. Jin et al., "Graphene nanonet for biological sensing applications," Nanotechnology, vol. 24, no. 37, Article ID 375302, 2013.

[3] Y. Wang, Z. Li, J. Wang, J. Li, and Y. Lin, "Graphene and graphene oxide: biofunctionalization and applications in biotechnology," Trends in Biotechnology, vol. 29, no. 5, pp. 205212, 2011.

[4] F. Schedin, A. K. Geim, S. V. Morozov et al., "Detection of individual gas molecules adsorbed on graphene," Nature Materials, vol. 6, no. 9, pp. 652-655, 2007.

[5] H. Choi, J. S. Choi, J.-S. Kim et al., "Flexible and transparent gas molecule sensor integrated with sensing and heating graphene layers," Small, vol. 10, no. 18, pp. 3685-3691, 2014.

[6] A. Girdhar, C. Sathe, K. Schulten, and J.-P. Leburton, "Graphene quantum point contact transistor for DNA sensing," Proceedings of the National Academy of Sciences of the United States of America, vol. 110, no. 42, pp. 16748-16753, 2013.

[7] T. Morita and S. Lindsay, "Determination of single molecule conductances of alkanedithiols by conducting-atomic force microscopy with large gold nanoparticles," Journal of the American Chemical Society, vol. 129, no. 23, pp. 7262-7263, 2007.

[8] X. D. Cui, A. Primak, X. Zarate et al., "Reproducible measurement of single-molecule conductivity," Science, vol. 294, no. 5542, pp. 571-574, 2001.

[9] B. Xu and N. J. Tao, "Measurement of single-molecule resistance by repeated formation of molecular junctions," Science, vol. 301, no. 5637, pp. 1221-1223, 2003.

[10] L. Venkataraman, J. E. Klare, C. Nuckolls, M. S. Hybertsen, and M. L. Steigerwald, "Dependence of single-molecule junction conductance on molecular conformation," Nature, vol. 442, no. 7105, pp. 904-907, 2006.

[11] S. Y. Quek, L. Venkataraman, H. J. Choi, S. G. Louie, M. S. Hybertsen, and J. B. Neaton, "Amine-gold linked singlemolecule circuits: experiment and theory," Nano Letters, vol. 7, no. 11, pp. 3477-3482, 2007.

[12] M. T. González, E. Leary, R. García et al., "Break-junction experiments on acetyl-protected conjugated dithiols under different environmental conditions," The Journal of Physical Chemistry C, vol. 115, no. 36, pp. 17973-17978, 2011.

[13] M. T. González, X. Zhao, D. Z. Manrique et al., "Structural versus electrical functionalization of oligo(phenylene ethynylene) diamine molecular junctions," The Journal of Physical Chemistry C, vol. 118, no. 37, pp. 21655-21662, 2014.

[14] S. Kaneko, T. Nakazumi, and M. Kiguchi, "Fabrication of a welldefined single benzene molecule junction using Ag electrodes," The Journal of Physical Chemistry Letters, vol. 1, no. 24, pp. 35203523, 2010.

[15] B. Xu, X. Xiao, and N. J. Tao, "Measurements of single-molecule electromechanical properties," Journal of the American Chemical Society, vol. 125, no. 52, pp. 16164-16165, 2003.

[16] A. Batra, P. Darancet, Q. Chen et al., "Tuning rectification in single-molecular diodes," Nano Letters, vol. 13, no. 12, pp. 62336237, 2013.

[17] S. Y. Quek, M. Kamenetska, M. L. Steigerwald et al., "Mechanically controlled binary conductance switching of a singlemolecule junction," Nature Nanotechnology, vol. 4, no. 4, pp. 230-234, 2009.

[18] A. Majumdar, "Thermoelectricity in semiconductor nanostructures," Science, vol. 303, no. 5659, pp. 777-778, 2004.

[19] M. Galperin and A. Nitzan, "Molecular optoelectronics: the interaction of molecular conduction junctions with light," Physical Chemistry Chemical Physics, vol. 14, no. 26, pp. 94219438, 2012.

[20] Q. Su, D. Wesner, H. Schönherr, and G. Nöll, "Molecular beacon modified sensor chips for oligonucleotide detection with optical readout," Langmuir, vol. 30, no. 47, pp. 14360-14367, 2014.

[21] W. Tan, X. Ruan, Q. Yu, Z. Yu, and X. Huang, "Fabrication of a $\mathrm{SnO}_{2}$-based acetone gas sensor enhanced by molecular imprinting," Sensors, vol. 15, no. 1, pp. 352-364, 2015.

[22] C. Ayela, G. Dubourg, C. Pellet, and K. Haupt, "All-organic microelectromechanical systems integrating specific molecular recognition-a new generation of chemical sensors," Advanced Materials, vol. 26, no. 33, pp. 5876-5879, 2014.

[23] M. Strange, K. S. Thygesen, J. P. Sethna, and K. W. Jacobsen, "Anomalous conductance oscillations and half-metallicity in atomic Ag-O chains," Physical Review Letters, vol. 101, no. 9, Article ID 096804, 2008. 
[24] W. H. A. Thijssen, D. Marjenburgh, R. H. Bremmer, and J. M. van Ruitenbeek, "Oxygen-enhanced atomic chain formation," Physical Review Letters, vol. 96, no. 2, Article ID 026806, 2006.

[25] A. I. Yanson, G. R. Bollinger, H. E. van den Brom, N. Agraï, and J. M. van Ruitenbeek, "Formation and manipulation of a metallic wire of single gold atoms," Nature, vol. 395, no. 6704, pp. 783-785, 1998.

[26] D. J. Mowbray, G. Jones, and K. S. Thygesen, "Influence of functional groups on charge transport in molecular junctions," The Journal of Chemical Physics, vol. 128, no. 11, Article ID 111103, 2008.

[27] M. Tsutsui, M. Taniguchi, and T. Kawai, "Quantitative evaluation of metal-molecule contact stability at the single-molecule level," Journal of the American Chemical Society, vol. 131, no. 30, pp. 10552-10556, 2009.

[28] M. Frei, S. V. Aradhya, M. S. Hybertsen, and L. Venkataraman, "Linker dependent bond rupture force measurements in singlemolecule junctions," Journal of the American Chemical Society, vol. 134, no. 9, pp. 4003-4006, 2012.

[29] S. Y. Quek, H. J. Choi, S. G. Louie, and J. B. Neaton, "Length dependence of conductance in aromatic single-molecule junctions," Nano Letters, vol. 9, no. 11, pp. 3949-3953, 2009.

[30] J. A. Malen, P. Doak, K. Baheti, T. D. Tilley, R. A. Segalman, and A. Majumdar, "Identifying the length dependence of orbital alignment and contact coupling in molecular heterojunctions," Nano Letters, vol. 9, no. 3, pp. 1164-1169, 2009.

[31] M. L. Perrin, F. Prins, C. A. Martin et al., "Influence of the chemical structure on the stability and conductance of porphyrin single-molecule junctions," Angewandte Chemie, vol. 50, no. 47, pp. 11419-11422, 2011.

[32] S. Martin, W. Haiss, S. J. Higgins, and R. J. Nichols, "The impact of E-Z photo-isomerization on single molecular conductance," Nano Letters, vol. 10, no. 6, pp. 2019-2023, 2010. 

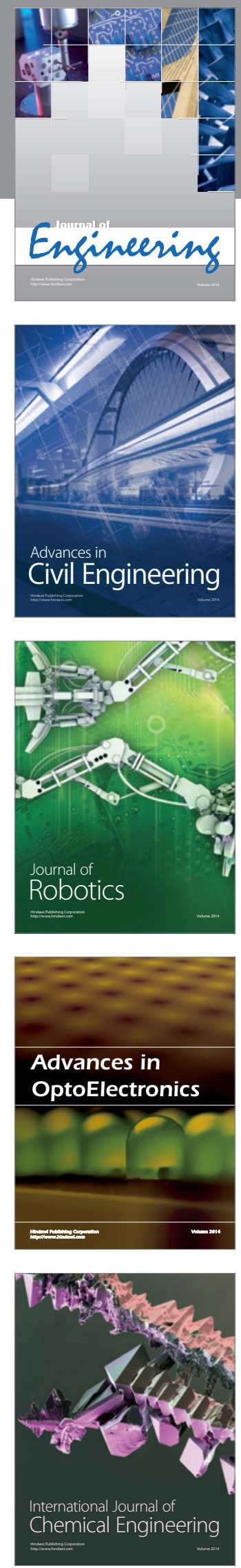

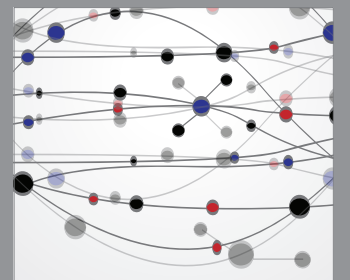

The Scientific World Journal
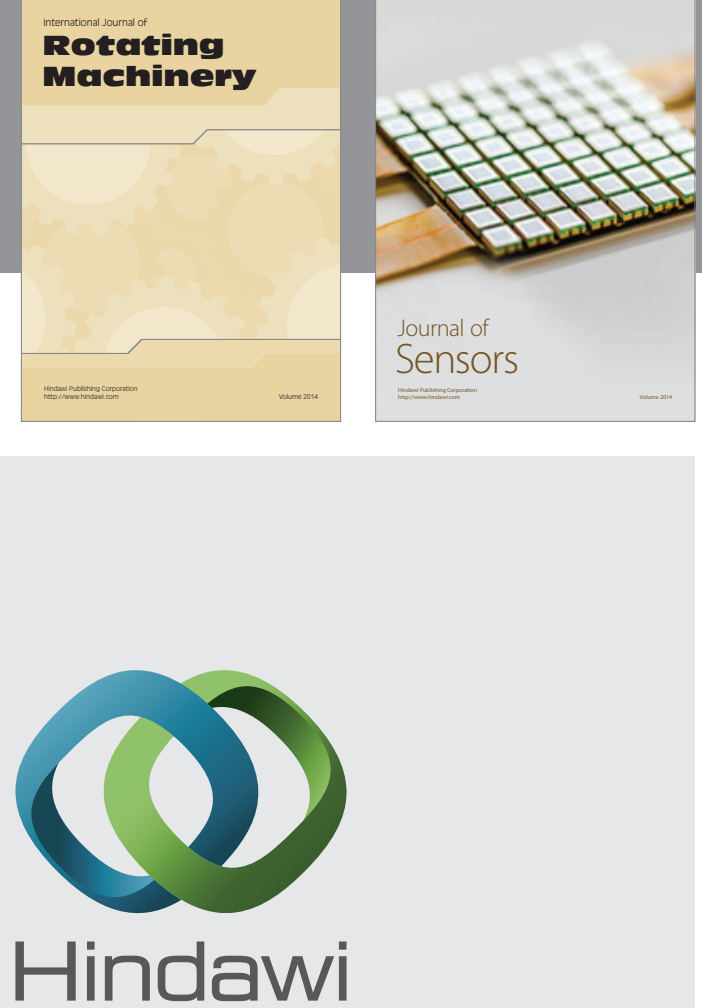

Submit your manuscripts at http://www.hindawi.com
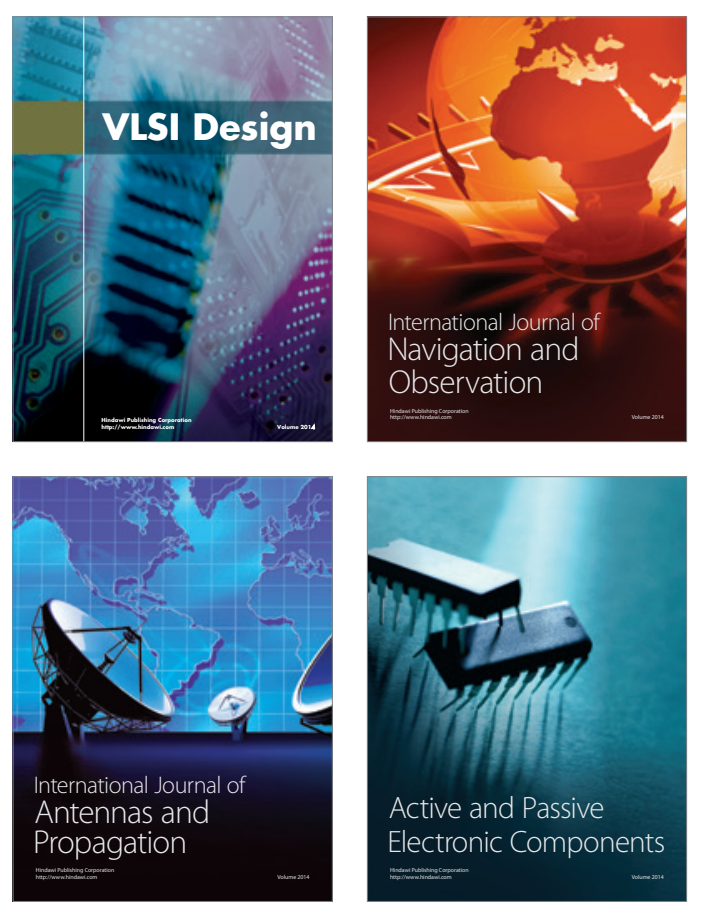
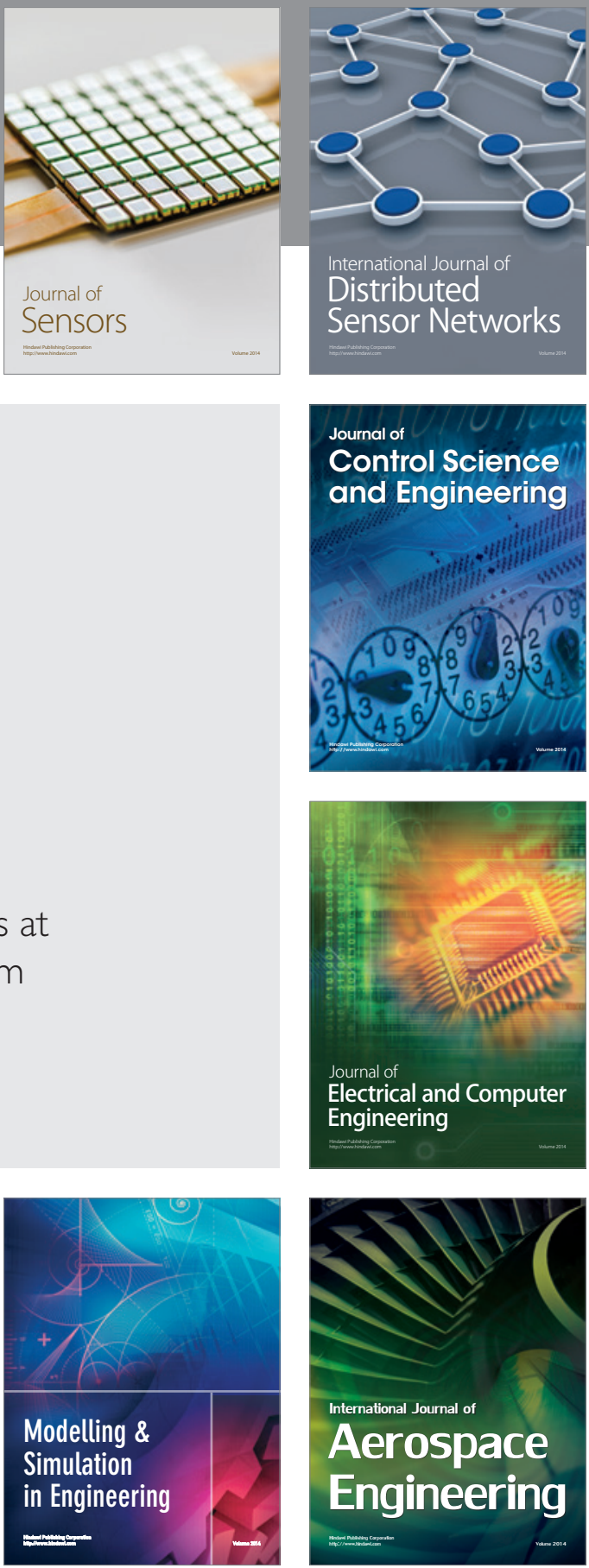

Journal of

Control Science

and Engineering
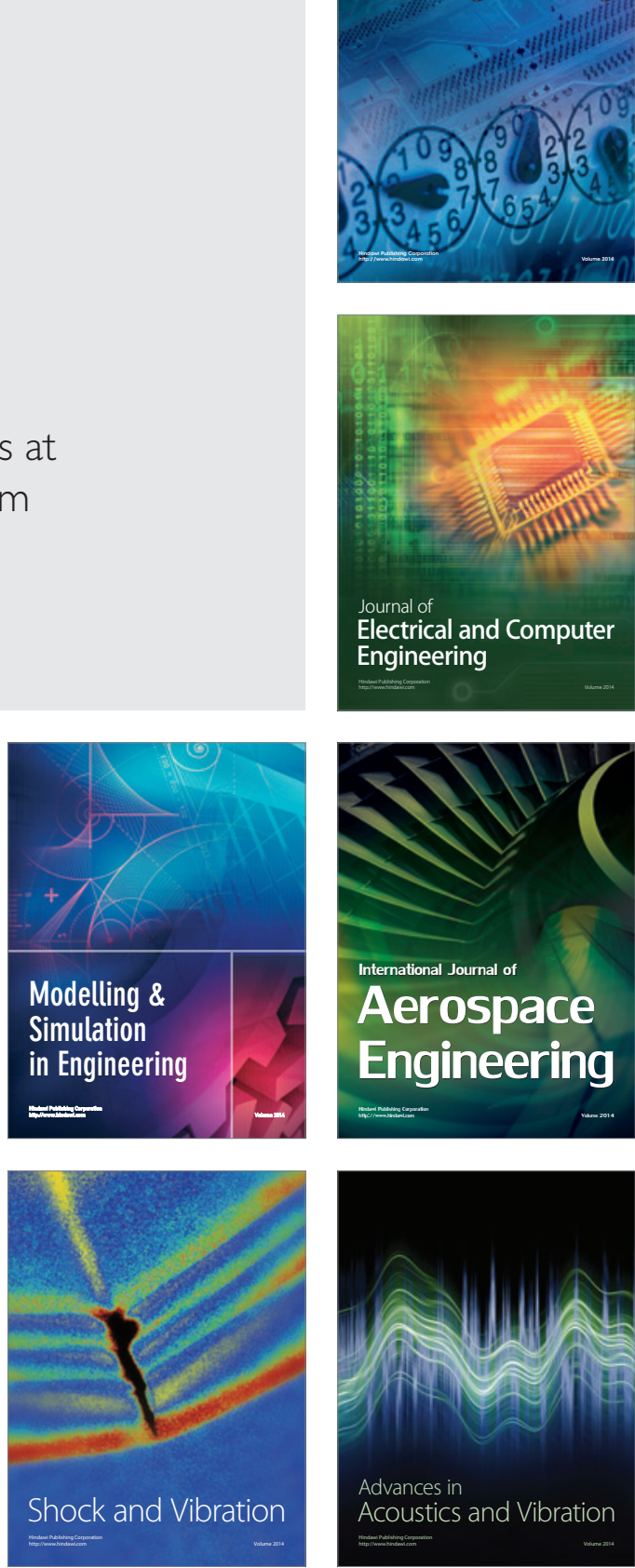